\title{
WestVirginiaUniversity
}

THE RESEARCH REPOSITORY @ WVU

Graduate Theses, Dissertations, and Problem Reports

2007

\section{State minimization problems in finite state automata}

Chris Tauras

West Virginia University

Follow this and additional works at: https://researchrepository.wvu.edu/etd

\section{Recommended Citation}

Tauras, Chris, "State minimization problems in finite state automata" (2007). Graduate Theses, Dissertations, and Problem Reports. 2541.

https://researchrepository.wvu.edu/etd/2541

This Thesis is protected by copyright and/or related rights. It has been brought to you by the The Research Repository @ WVU with permission from the rights-holder(s). You are free to use this Thesis in any way that is permitted by the copyright and related rights legislation that applies to your use. For other uses you must obtain permission from the rights-holder(s) directly, unless additional rights are indicated by a Creative Commons license in the record and/ or on the work itself. This Thesis has been accepted for inclusion in WVU Graduate Theses, Dissertations, and Problem Reports collection by an authorized administrator of The Research Repository @ WVU. For more information, please contact researchrepository@mail.wvu.edu. 


\title{
State Minimization Problems in Finite State Automata
}

\author{
by \\ Chris Tauras \\ Thesis submitted to the \\ College of Engineering and Mineral Resources \\ at West Virginia University \\ in partial fulfillment of the requirements \\ for the degree of \\ Master of Science \\ in \\ Computer Science
}

K. Subramani, Ph.D., Chair

A. Ross, Ph.D.

B. Cukic, Ph.D.

Lane Department of Computer Science and Electrical Engineering

Morgantown, West Virginia

2007

Keywords: Finite Automata, State Minimization

Copyright 2007 Chris Tauras 


\author{
Abstract \\ State Minimization Problems in Finite State Automata \\ by \\ Chris Tauras \\ Master of Science in Computer Science \\ West Virginia University \\ K. Subramani, Ph.D., Chair
}

In this thesis, we analyze the problem of state minimization in 2-MDFAs. The class of 2-MDFAs is an extension of the class of DFAs, allowing a small amount of nondeterminism; specifically two start states. Since nondeterminism allows finite automata to be more succinct, it is worthwhile to investigate the problem of minimizing such finite automata. In the case of unbounded non- determinism, i.e., NFAs, such automata can be exponentially more succinct than DFAs [1], but the corresponding minimization problem is PSPACE-complete [2]. Even in the case of 2-MDFAs, which are only polynomially more succinct than DFAs, the minimization problem remains non-trivial; indeed, [3] shows that the corresponding decision problem is NPcomplete. We are concerned with the approximability of the 2-MDFA minimization problem. Our main contribution in the current work is the design of an n-factor approximation algorithm for state minimization in 2-MDFAs. 


\section{Chapter 1}

\section{Introduction}

Finite Automata (FAs) are used in many applications such as lexical analysis, parsing, and hashing. As acceptance of a particuliar string can be easily tested in polynomial time, it is desirable to represent the finite automata using the minimum amount of space. A regular language can be represented as a Deterministic Finite Automaton (DFA), a Multiple Start State DFA (MDFA) or as a Non-deterministic Finite Automaton (NFA). While each type of finite automaton represents exactly the class of regular languages, their relative succinctness and the complexities of their minimization problems vary greatly. NFAs can be exponentially more succinct than DFAs [1], but the corresponding minimization problem is PSPACE-complete [2], in contrast to polynomial-time [4]. MDFAs represent an interesting type of finite automata that have recently received much attention in the literature. These FAs have a limited amounted of non-determinism in that there are multiple start states; however, there is precisely one target state for each state on a given input. Even this small amount of nondeterminism in 2-MDFAs is enough to result in a hard minimiztion problem; in particuliar, the problem of state minimization is NP-complete for 2-MDFAs. [3]. Unlike general NFAs, which can be exponentially more succinct than DFAs [1], 2-MDFAs are only quadratically more succinct than DFAs (See Section 4). Inasmuch as the state minimization problem in 2-MDFAs is in NP, it is worthwhile to ask whether there exists a polynomial time bounded-error approximation algorithm for this problem. We answer this question in the affirmative, by presenting and analyzing a linear factor approximation algorithm for the same.

The rest of this thesis is organized as follows: Section 2 contains a formal description of the state minimization problem in 2-MDFAs. Section 3 describes the motivation for our work as well as related approaches in the literature. Section 4 describes an $n$-approximation algo- 
rithm for the state minimization problem in 2-MDFAs; as part of the analysis, an algorithm is described for converting a 2-MDFA into a DFA. This section also includes a detailed analysis of the approximation bound. In Section 5, a number of problems are proposed, which are related to the 2-MDFA state minimization problem and provide motivation for the same. Section 6 provides a detailed implementation profile of our algorithm over a wide range of inputs. We conclude in Section 7 by summarizing the main results and detailing problems for future research. 


\section{Chapter 2}

\section{Statement of Problem}

Formally, a 2-MDFA $M$ is defined by the 6 -tuple $\left\langle Q_{M}, \Sigma, \delta_{M}, q_{s}^{0 M}, q_{s}^{1 M}, F_{M}\right\rangle$, in which:

(a) $Q_{M}$ denotes the set of states. $\left|Q_{M}\right|$ denotes the state complexity of $M$.

(b) $\Sigma$ is the input alphabet. Without loss of generality, it is assumed that the alphabet $\Sigma=$ $\{0,1\}$ for all the finite automata discussed in the remainder of this thesis.

(c) $\delta_{M}$ is the transition function that maps each pair $\langle q, a\rangle \in Q_{M} \times \Sigma$ to a state $q \in Q_{M}$, i.e., $\delta: Q_{M} \times \Sigma \rightarrow Q_{M}$.

(d) $q_{s}^{0 M}$ and $q_{s}^{1 M}$ are the two start states.

(e) $F_{M}$ is the set of final states.

Figure 2.1 shows an example of a 2-MDFA. It accepts the language $(01)^{*} 0 \cup(10)^{*} 1 \cup$ $(01)^{*} 00(01)^{*}$.

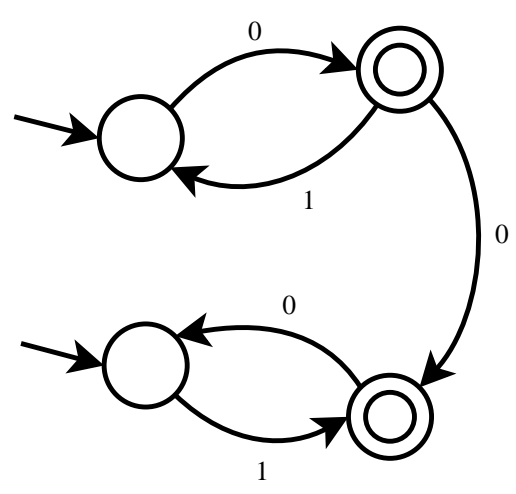

Figure 2.1: An example 2-MDFA $M_{0}=M_{s}$ 
As with DFAs, we extend $\delta$ to $\hat{\delta}$, where $\hat{\delta}$ is a function mapping tuples of states and strings to states. Formally, $\hat{\delta}: Q_{M} \times \Sigma^{*} \rightarrow Q_{M}$ is defined recursively as follows:

(a) $\forall q \in Q_{M}, \hat{\delta}(q, \epsilon)=q$.

(b) $\forall\langle q, a, w\rangle \in Q_{M} \times \Sigma \times \Sigma^{*}, \hat{\delta}(q, w a)=\delta(\hat{\delta}(q, w), a)$.

Definition 2.1 A string $x \in \Sigma^{*}$ is said to be accepted by the 2-MDFA $M$, if and only if $\left(\left\{\hat{\delta}\left(q_{s}^{0 M}, x\right)\right\} \cup\left\{\hat{\delta}\left(q_{s}^{1 M}, x\right)\right\}\right) \cap F_{M} \neq \phi$.

Definition 2.2 The language of a 2-MDFA $M$, denoted by $L(M)$, is the set of all strings $x \in \Sigma^{*}$ which are accepted by $M$.

Given a 2-MDFA $M=\left\langle Q_{M}, \Sigma, \delta_{M}, q_{s}^{0 M}, q_{s}^{1 M}, F_{M}\right\rangle$, we define $L\left(q_{s}^{0 M}\right)$ to be the set of of strings $x \in \Sigma^{*}$, such that $\left\{\hat{\delta}_{M}\left(q_{s}^{0 M}, x\right)\right\} \cap F_{M} \neq \phi$. In other words, $L\left(q_{s}^{0 M}\right)$ is the set of strings that are accepted by $M$, under the provision that the start state is $q_{s}^{0 M}$. The language $L\left(q_{s}^{1 M}\right)$ is analogously defined. It is not hard to see that $L(M)=L\left(q_{s}^{0 M}\right) \cup L\left(q_{s}^{1 M}\right)$.

Definition 2.3 Two 2-MDFAs $M_{1}$ and $M_{2}$ are equivalent if and only if $L\left(M_{1}\right)=L\left(M_{2}\right)$.

Definition 2.4 An $\epsilon$-NFA is a nondeterministic finite automaton that has the additional ability to transition without consuming the next input character. These spontaneous transitions are known as $\epsilon$-transitions. The addition of this ability does not allow acceptance of any nonregular languages.

Regardless of the type of automaton $M$ under consideration, we use $L(M)$ to denote the language represented by that automaton and the phrase state complexity to denote the number of states $Q_{M}$.

Now that all the necessary preliminaries are covered, the problem may now be formally stated:

$\mathbf{P}_{1}$ : Given a 2-MDFA $M_{1}=\left\langle Q_{M_{1}}, \Sigma, \delta_{M_{1}}, q_{s}^{0 M_{1}}, q_{s}^{1 M_{1}}, F_{M_{1}}\right\rangle$ and a number $K$, is there a 2-MDFA $M_{2}=\left\langle Q_{M_{2}}, \Sigma, \delta_{M_{2}}, q_{s}^{0 M_{2}}, q_{s}^{1 M_{2}}, F_{M_{2}}\right\rangle$, such that $L\left(M_{1}\right)=L\left(M_{2}\right)$ and $\left|Q_{M_{2}}\right| \leq K$ ? 


\section{Chapter 3}

\section{Motivation and Related Work}

The motivation for the study of finite automata comes from many applications, including lexical analysis, hashing, pattern matching, and, of course, the syntax of programming languages [5]. In the case of lexical analysis, compilers for high-level languages, such as Fortran or $\mathrm{C}$, often implement a finite automaton for the lexical analysis for their first pass. Such a finite automaton may possess hundreds or even thousands of states. The large number of states that are potentially used translates to a large amount of memory required by the compiler. Therefore, a method for reducing the number of states would be beneficial in saving space, reducing the cost of implementation. In the case of hashing, the data to be hashed is given as an input to a hashing DFA. The state that the DFA is in, at the end of the string, is the hash value. One way to use a 2-MDFA instead of a DFA for hashing is to use a pair of states after computation, say one state for the high bits and one for the low bits, instead of a single state for the complete value (For instance, see Figure 3.1). The implementation of a 2-MDFA would allow a more complex automaton to be implemented while simultaneously reducing the space requirements. In the case of pattern matching, when strings are concerned, regular expressions are often used to define the desired pattern to match or to search for. On the account of the connection between regular expressions and finite automata, algorithms to do this often make use of finite automata to perform the search or match operation, such as in [6] and [7]. It should be noted that automata with more states require more space to implement, so it is desirable to reduce the number of states needed.

The problem of state minimization has been studied extensively in a number of different types of finite automata. It is well-known that the problem of minimizing DFAs is solvable in polynomial time [4], whereas the problem of minimizing NFAs is PSPACE-complete [2]. 


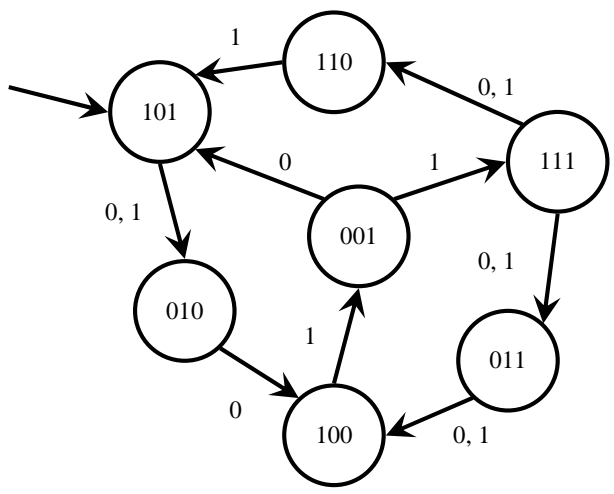

Figure 3.1: Using finite automata in hashing

In an effort to determine how much nondeterminism can be permitted in finite automata while keeping the state minimization problem easy, several classes of finite automata with limited nondeterminism have been studied. Unambiguous Finite State Automata (UFAs) form one such class; for any input string, there is never more than one accepting path. The problem of minimizing the number of states in a UFA has been shown to be NP-complete [1], as well as converting a DFA to a minimal UFA [8]. In [3], the complexity of state minimization problems in $k$-MDFAs was investigated and shown to be NP-complete for $k \geq 2$, where $k$ denotes the number of distinct start states in the MDFA. We focus on the problem on minimizing the number of states in a 2-MDFA, with a view towards obtaining non-trivial approximation bounds; additionally, the the degree of succinctness of 2-MDFAs is explored. The complexity inherent in representing regular languages succinctly has been studied in [9, 10] and [11]. [12] relates the type of ambiguity of finite automata to the succinctness of their representation. We thus see that determining succinct (minimal) representations of languages is of enormous interest to the Automata Theory community.

The difficulty of dealing with NP-Hard optimization problems can be mitigated to some extent by devising efficient approximation algorithms [13]. Basically, a polynomial time approximation algorithm for an optimization (minimization) problem delivers an output in polynomial time, but with a certain loss of accuracy. Such algorithms are useful in applications in which accuracy can be sacrificed to some extent, if the output is computed quickly. The relevance of these algorithms and their practical impact are discussed at length in [13].

Definition 3.1 Let $\Pi$ denote a minimization problem, and let OPT denote an optimal algorithm for the same. For an arbitrary instance $I \in \Pi, O P T(I)$ refers to the output of OPT. Let 
$\mathcal{A}$ denote an algorithm for $\Pi$, with $\mathcal{A}(I)$ denoting the output of algorithm $\mathcal{A}$ on an arbitrary instance $I \in \Pi$. $\mathcal{A}$ is said to be a polynomial time approximation algorithm for $\Pi$, if:

(a) $\mathcal{A}$ runs in polynomial time.

(b) For all instances $I \in \Pi, \mathcal{A}(I) \leq c \cdot O P T(I)$, where $c$ is called the factor of approximation.

Provided that the above conditions are met, algorithm $\mathcal{A}$ is said to be a $c$-factor approximation algorithm for the problem $\Pi$.

The analysis of an approximation algorithm typically proceeds in two steps. First it is shown that for all instances $I \in \Pi, \mathcal{A}(I) \leq c_{1}$; then it is shown that for all instances $I \in$ $\Pi, O P T(I) \geq c_{2}$. From these two proofs, we can conclude that for all instances $I \in \Pi$, $\mathcal{A}(I) \leq \frac{c_{1}}{c_{2}} \cdot O P T(I)$. It is important to note that our analysis is significantly different from the traditional analyses of approximation algorithms.

Within the field of Automata theory, there has been no active effort to study approximation algorithms as a tool; indeed we could find only one result [14], and that result was concerned with the inapproximability of the Minimum Consistent Finite Automaton problem [15].

Our work establishes that there exists an $n$-factor approximation algorithm for problem $\mathbf{P}_{\mathbf{1}}$, where $n$ denotes the minimum number of states required to represent the input language as a 2-MDFA. 


\section{Chapter 4}

\section{Algorithm and Analysis}

We shall now present our algorithm for approximating the minimum number of states required to represent the input language as a 2-MDFA. At the heart of our algorithm is a procedure that converts a 2-MDFA into a DFA. We shall argue that the conversion results in only a quadratic increase in the number of states; this is in contrast to the procedure which converts an NFA into a DFA, in which the state blowup could be exponential.

Our algorithm proceeds as follows: First, the input 2-MDFA $M_{0}$ is pruned; the pruning procedure consists of eliminating redundant and unreachable states. The details of the procedure are described in SHRINK-2MDFA() (Algorithm 4.3). The resultant 2-MDFA $M_{s}$ is then converted into an $\epsilon$-NFA $M_{1}^{\prime}$. Subsequently, $M_{1}^{\prime}$ is converted into an NFA $M_{2}$ and then into a DFA $M_{3}$. This is followed by $M_{3}$ being minimized to obtain $M_{4}$. Finally, we return either $M_{s}$ or $M_{4}$, depending on which of the two has smaller state complexity.

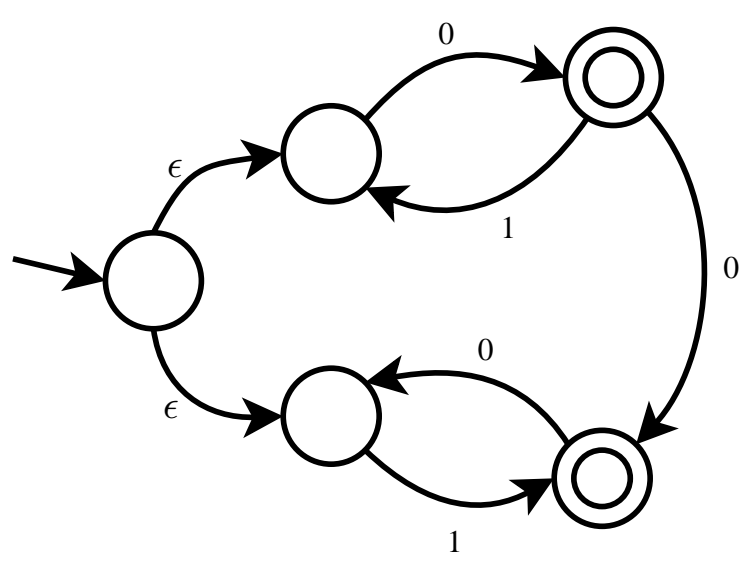

Figure 4.1: $M_{1}^{\prime}$ 


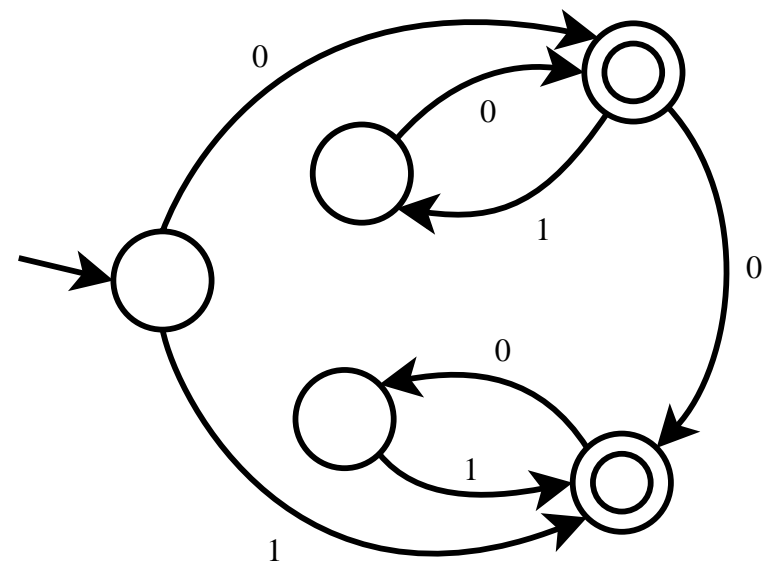

Figure 4.2: $M_{2}=M_{3}=M_{4}$

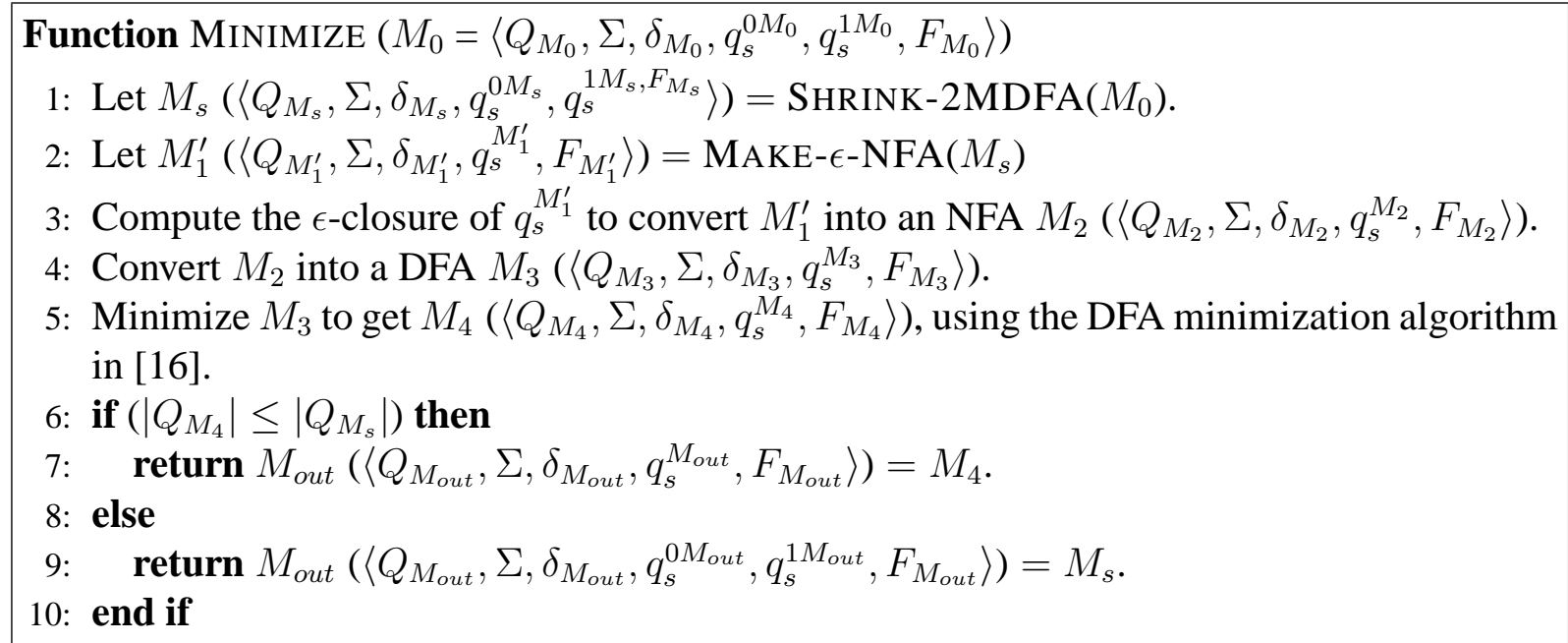

Algorithm 4.1: 2-MDFA Minimization

Function MAKE- $\epsilon$-NFA $\left(M_{s}=\left\langle Q_{M_{s}}, \Sigma, \delta_{M_{s}}, q_{s}^{0 M_{s}}, q_{s}^{1 M_{s}}, F_{M_{s}}\right\rangle\right)$

1: Insert a new start state $q_{s}^{M_{s}}$ into $M_{s}$.

2: Insert $\epsilon$-transitions from $q_{s}^{M_{s}}$ to $q_{s}^{0 M_{s}}$ and $q_{s}^{1 M_{s}}$.

3: $\left\{q_{s}^{0 M_{s}}\right.$ and $q_{s}^{1 M_{s}}$ are no longer start states. $\}$

4: return $M_{s}$

Algorithm 4.2: Conversion to $\epsilon$-NFA 
The purpose of the SHRINK-2MDFA() function is to eliminate useless states from the input 2-MDFA.

Definition 4.1 Two states $p$ and $q$ are said to be equivalent in a finite automaton $M$, if, for all strings $w \in \Sigma^{*}, \hat{\delta}_{M}(p, w)$ leads to an accepting state in $M$ if and only if $\hat{\delta}_{M}(q, w)$ does.

Any pair of equivalent states $p$ and $q$ may be merged without affecting the language of the finite automaton $M$ in question. This is because the remainder of the input string $w$ will either be accepted by both states or be rejected by both states.

Definition 4.2 A state $p$ in a 2-MDFA $M_{0}$ is said to be reachable if there is a directed path from either $q_{s}^{0 M_{0}}$ or $q_{s}^{1 M_{0}}$ to $p$ in the directed graph representing $M_{0}$.

A state which is not reachable from either start state is called unreachable. Clearly, such states cannot possibly be part of the computational path for any input string, so the language of the 2-MDFA in question will not change if such states are removed.

Function SHRINK-2MDFA $\left(M_{0}=\left\langle Q_{M_{0}}, \Sigma, \delta_{M_{0}}, q_{s}^{0 M_{0}}, q_{s}^{1 M_{0}}, F_{M_{0}}\right\rangle\right)$

1: Let $M_{1}$ denote a copy of $M_{0}$

2: Replace each block of equivalent states with a single state, using the algorithm in [16].

3: Delete all unreachable states from $M_{1}$.

4: return $M_{1}$.

\section{Algorithm 4.3: Shrinking a 2-MDFA}

For instance, let the 2-MDFA represented by Figure 2.1 be the input $M_{0}$ to Algorithm 4.2. Since $M_{0}$ has no pairs of equivalent states and no unreachable states, the pruning procedure does not alter it and $M_{s}=M_{0}$. Figure 4.1 displays the 2-MDFA after it has been converted to an $\epsilon$-NFA $M_{1}^{\prime}$. $M_{1}^{\prime}$ is then converted to the NFA $M_{2}$ as shown in Figure 4.2. It so happens that $M_{2}$ is already in minimized DFA form, and, therefore, no further work needs to be done. Finally, $M_{s}$ has fewer states than $M_{4}$ and, hence, $M_{s}$ is returned by Algorithm 4.2.

Observation 4.1 Consider the NFA $M_{2}=\left\langle Q_{M_{2}}, \Sigma, \delta_{M_{2}}, q_{s}^{M_{2}} F_{M_{2}}\right\rangle$ in Line 4 of Algorithm 4.1. Observe that converting the $\epsilon-N F A M_{1}^{\prime}$ to an NFA $M_{2}$ involves the following steps: Compute the $\epsilon$-closure $S$ of $q_{s}^{M_{1}^{\prime}}$; observe that $S$ contains $q_{s}^{M_{1}^{\prime}}$ and the two states, say $q_{r}$ and $q_{t}$, to which $M_{1}^{\prime}$ can move from $q_{s}^{M_{1}^{\prime}}$ on $\epsilon$-transitions. Thus, it is clear that $|S|=3 . q_{s}^{M_{1}^{\prime}}$ is replaced with a new start state $q_{s}^{M_{2}}$. For each symbol $a \in \Sigma$, we set $\delta\left(q_{s}^{M_{2}}, a\right)=\delta\left(q_{r}^{M_{1}^{\prime}}, a\right) \cup \delta\left(q_{t}^{M_{1}^{\prime}}, a\right)$. 
The remaining states and transitions are identical in $M_{1}^{\prime}$ and $M_{2}$. Note that this construction exploits the special structure of $M_{1}^{\prime}$. For any symbol $a \in \Sigma$, we observe that $\left|\delta_{M_{2}}\left(q_{s}^{M_{2}}, a\right)\right| \leq 2$, whereas, for all other states $q \in Q_{M_{2}},\left|\delta_{M_{2}}(q, a)\right| \leq 1$. In other words, the non-determinism of $M_{2}$ is limited to the first move.

Observation 4.2 There does not exist a directed path from any state in the $\epsilon$-NFA $M_{1}^{\prime}$ to the start state $q_{s}^{M_{1}^{\prime}}$. Therefore, it follows that the start state $q_{s}^{M_{2}}$ of $M_{2}$ is not reachable from any other state in $M_{2}$.

Lemma 4.1 For any string $x \in \Sigma^{*},\left|\hat{\delta}_{M_{2}}\left(q_{s}^{M_{2}}, x\right)\right| \leq 2$.

Proof. We prove Lemma 4.1 by using induction on the length of the input string $x$.

BASE CASE: $|x|=0$ and, hence, $x=\epsilon$. Since $x=\epsilon$, it follows that $M_{2}$ does not make any move and stays in the start state. In other words, $\left|\hat{\delta}_{M_{2}}\left(q_{s}^{M_{2}}, x\right)\right|=1 \leq 2$.

INDUCTIVE STEP: Assume that $\left|\hat{\delta}_{M_{2}}\left(q_{s}^{M_{2}}, y\right)\right| \leq 2$ whenever $M_{2}$ is presented with a string $y$ such that $|y| \leq n-1$. Now consider a string, $x=w a$, with $|x|=n,|w|=n-1$ and $a \in \Sigma$. Let us define $Q_{\delta}=\hat{\delta}_{M_{2}}\left(q_{s}^{M_{2}}, w\right)$. As per the inductive hypothesis, $\left|Q_{\delta}\right| \leq 2$. Without loss of generality, let us assume that $Q_{\delta}$ consists of exactly two states, say $q_{u}$ and $q_{v}$. Note that transitions out of $q_{u}$ and $q_{v}$ are deterministic, i.e. $\left|\hat{\delta}_{M_{2}}\left(q_{u}, a\right)\right|=1$ and $\left|\hat{\delta}_{M_{2}}\left(q_{v}, a\right)\right|=1$. Therefore, $\left|\hat{\delta}_{M_{2}}\left(q_{s}^{M_{2}}, x\right)\right|=\left|Q_{\delta}\right| \leq 2$, and the claim follows.

Lemma 4.2 The number of states in $M_{3}$ is $O\left(\left|Q_{M_{s}}\right|^{2}\right)$.

Proof. Note that the general algorithm for converting an NFA to a DFA enumerates all possible subsets of states in which the NFA can exist. In general, there is an exponential number of subsets to enumerate. However, in this case, the NFA $M_{2}$ can exist in at most two states after reading an input string; consequently, the conversion algorithm need only enumerate those subsets that contain no more than two states. Hence, the number of states that need to be enumerated is $O\left(\left|Q_{s}\right|^{2}\right)$, which also represents the state complexity of $M_{3}$.

Theorem 4.1 Algorithm 4.1 runs in time polynomial in the size of its input.

Proof. Each step of Function Minimize() can be implemented to run in time that is polynomial in the size of the input. It is resonable to consider the number of states of the input 2-MDFA the "size" $n$ of the input. Although the representation of each state technically needs $O(\log n)$ 
bits, and although the transition function needs to be included in the input, these things would only result in an input size of $O\left(n^{2} \log n\right)$ bits, so, for simplicity, these things may be ignored.

Line 1 involves eliminating blocks of equivalent states and can be implemented in $O\left(n^{3}\right)$ time, as per the algorithm in [16]. The conversion of $M_{s}$ into an $\epsilon$-NFA $M_{1}^{\prime}$ is a constant time operation with the addition of one state; likewise, as discussed above, the conversion of $M_{1}^{\prime}$ into an NFA $M_{2}$ is a constant time operation. Line 4 involves the conversion of the NFA $M_{2}$ into a DFA $M_{3}$; since we have to enumerate state pairs only, this operation can be implemented in $O\left(n^{3}\right)$ time. Note that $M_{3}$ has $O\left(n^{2}\right)$ states; it follows that the DFA minimization procedure in Line 5 can be implemented in time $O\left(n^{6}\right)$ time [16].

Thus, Algorithm 4.1 can be implemented in time that is polynomial in the size of its input.

Theorem 4.2 Let $L\left(M_{0}\right)$ denote the regular language represented by the input 2-MDFA $M_{0}$ and let $n=\left|Q_{M_{4}}\right|$ denote the number of states in the minimized 2-MDFA $M_{4}$ (See Algorithm 4.1). The optimal 2-MDFA for $M_{1}$, i.e., the 2-MDFA with the fewest number of states representing $L\left(M_{0}\right)$, must have $\Omega\left(n^{\frac{1}{2}}\right)$ states.

Proof. Let $S_{E M_{0}}$ denote the set of all 2-MDFAs that are equivalent to the input 2-MDFA $M_{0}$. The minimum state DFA corresponding to a given language $L\left(M_{0}\right)$ is unique [16]; accordingly, regardless of the 2-MDFA in $S_{E M_{0}}$ that is presented as input to Algorithm 4.1, the number of states in the minimized DFA $M_{4}$ is the same. Let $M_{o p t}=\left\langle Q_{M_{o p t}}, \Sigma, \delta_{M_{o p t}}, q_{s}^{0 M_{o p t}}, q_{s}^{1 M_{o p t}}, F_{M_{o p t}}\right\rangle$ denote the optimal 2-MDFA corresponding to $L\left(M_{0}\right)$ Clearly, $M_{o p t} \in S_{E M_{0}}$; further, $\left|Q_{o p t}\right| \leq$ $\left|Q_{M_{r}}\right|, \forall M_{r} \in S_{E M_{0}}$. Let us focus on the situation in which $M_{o p t}$ is presented to Algorithm 4.1. The corresponding $M_{4}$ would be no larger than $O\left(\left|Q_{M_{o p t}}\right|^{2}\right)$ as per the discussion above. But $M_{4}$ and, therefore, $\left|Q_{M_{4}}\right|$ are the same for all 2-MDFAs $M_{r} \in S_{E M_{0}}$; note that this includes $M_{0}$. Therefore, it follows that the output of Algorithm 4.1 on input $M_{0}$ has at most $O\left(\left|Q_{M_{o p t}}\right|^{2}\right)$ states. In other words, if the output of Algorithm 4.1 on an input has $n$ states, then the optimal 2-MDFA for this instance has $\Omega\left(n^{\frac{1}{2}}\right)$ states.

Corollary 4.1 Algorithm 4.1 is a linear factor approximation algorithm, where the linear factor refers to the size of the optimal 2-MDFA.

Proof. From Theorem 4.2 and the discussion in Section 3, it is clear that the output produced by Algorithm 4.1 is off by at most a linear factor from the optimum; in other words, if the state 
complexity of $M_{o p t}$ is $p$, then the state complexity of $M_{o p t}$, i.e., the automaton that is output by Algorithm 4.1, is at most $p^{2}$. The claim follows.

The following theorem will show that the linear factor bound is tight for Algorithm 4.1; thus any improvement in the approximation bound will require the development of new techniques.

Theorem 4.3 There exists a 2-MDFA $M_{0}$ such that the optimal 2-MDFA for $L\left(M_{0}\right)$ has state complexity $p$, while the output of Algorithm 4.1 on $M_{0}$ has state complexity $p^{2}$.

Proof. Let $M_{d 1}=\left\langle Q_{M_{d 1}}, \Sigma, \delta_{M_{d 1}}, q_{s}^{M_{d 1}}, F_{M_{d 1}}\right\rangle$ and $M_{d 2}=\left\langle Q_{M_{d 2}}, \Sigma, \delta_{M_{d 2}}, q_{s}^{M_{d 2}}, F_{M_{d 2}}\right\rangle$ denote two minimal DFAs representing the languages $L\left(M_{d 1}\right)$ and $L\left(M_{d 2}\right)$ respectively, such that:

(a) The minimal DFA $M_{d 3}=\left\langle Q_{M_{d 3}}, \Sigma, \delta_{M_{d 3}}, q_{s}^{M_{d 3}}, F_{M_{d 3}}\right\rangle$ representing the language $L\left(M_{d 1}\right) \cup L\left(M_{d 2}\right)$ has state complexity $\Theta\left(\left|Q_{M_{d 1}}\right| \cdot\left|Q_{M_{d 2}}\right|\right)$.

(b) $\left|Q_{M_{d 1}}\right|=\Theta\left(\left|Q_{M_{d 2}}\right|\right)$.

It is well-known that such languages exist; for instance, see [9], where the properties of such languages are discussed. The minimal 2-MDFA for the language $L\left(M_{d 3}\right)$ clearly has state complexity $O\left(\left|Q_{M_{d 1}}\right|\right)$. However, we can construct a 2-MDFA $M_{0}=\left\langle Q_{M_{0}}, \Sigma, \delta_{M_{0}}, q_{s}^{0 M_{0}}, q_{s}^{1 M_{0}}, F_{M_{0}}\right\rangle$ with the following properties:

(i) $L\left(q_{s}^{0 M_{0}}\right)=L\left(M_{d 3}\right)$.

(ii) $L\left(q_{s}^{1 M_{0}}\right)=\epsilon$.

Note that $L\left(M_{0}\right)=L\left(q_{s}^{0 M_{0}}\right) \cup L\left(q_{s}^{0 M_{0}}\right)=L\left(M_{d 3}\right)$. Secondly, the DFA with start state $q_{s}^{0 M_{0}}$ has a transition function which is identical to the transition function of the DFA $M_{d 3}$, i.e., the state complexity of $M_{0}$ is $\Theta\left(\left|Q_{M_{d 1}}\right| \cdot\left|Q_{M_{d 2}}\right|\right)=\Theta\left(\left|Q_{M_{d 1}}\right|^{2}\right)$.

Accordingly, the SHRINK-2MDFA() procedure leaves $M_{0}$ unaltered, and the DFA minimization procedure returns a DFA with $\Theta\left(\left|Q_{M_{d 1}}\right|^{2} \mid\right)$ states. Since both procedures return a 2MDFA with $\Theta\left(\left|Q_{M_{d 1}}\right|^{2} \mid\right)$ states, Algorithm 4.1 necessarily returns a 2-MDFA with $\Theta\left(\left|Q_{M_{d 1}}\right|^{2} \mid\right)$ states. However, the optimal 2-MDFA for the language $L\left(M_{d 3}\right)$ has $O\left(\left|Q_{M_{d 1}}\right|\right)$ states, and the theorem follows.

The next theorem shows that Algorithm 4.1 is optimal in certain cases. 
Theorem 4.4 There exists a regular language $L_{0}$, such that Algorithm 4.1 computes the optimal

2-MDFA when presented with any input 2-MDFA that accepts $L_{0}$.

Proof. Let $L_{0}=\Sigma^{*}$. The optimal DFA for $L_{0}$ has precisely one state. Since this DFA is unique, the state complexity of the automaton returned by Algorithm 4.1 is always 1, regardless of the state complexity of the 2-MDFA that is used to represent $L_{0}$.

We make the following observations about Algorithm 4.1:

(1) The state complexity of the output of Algorithm $4.1\left(M_{\text {out }}\right)$ is never greater than the state complexity of its input $\left(M_{0}\right)$. Note that the SHRINK-2MDFA() procedure does not increase state complexity and, hence, $\left|Q_{s}\right| \leq\left|Q_{0}\right|$. Algorithm 4.1 returns either $M_{s}$ or the minimized DFA $M_{4}$ depending on which automaton has smaller state complexity.

(2) It follows that Algorithm 4.1 is optimal when the minimal 2-MDFA is given as the input.

From the empirical perspective (see Section 6), it appears that for a given 2-MDFA $M_{0}$, the SHRINK-2MDFA() procedure returns a better quality of approximation than the DFA minimization procedure. This suggests that by merely computing $M_{s}$, a good approximation is generally obtained; however, the following lemma proves that this is not true in general.

Lemma 4.3 As an approximation to the optimal 2-MDFA, the state complexity of the output of the SHRINK-2MDFA() procedure is arbitrarily bad.

Proof. Let $M_{0}$ denote a 2-MDFA, with $L\left(q_{s}^{0 M_{0}}\right)$ representing an arbitrary language with an arbitrary DFA state complexity $m$. Let $L\left(q_{s}^{1 M_{0}}\right)=\Sigma^{*}$. Note that the minimal 2-MDFA for $M_{0}$ has only one state that transitions to itself on all strings $x \in \Sigma^{*}$. When $M_{s}$ is computed from $M_{0}$ using the SHRINK-2MDFA() procedure, the language of the 2-MDFA start states is not redefined, and, hence, $L\left(q_{s}^{0 M_{0}}\right)$ and $L\left(q_{s}^{1 M_{0}}\right)$ remain the same. Therefore, $M_{s}$ has state complexity $\Theta(m)$, which is arbitrarily bad as an approximation.

The principal drawback of the SHRINK-2MDFA() procedure is that it does not alter the languages of the individual start states. 


\section{Chapter 5}

\section{Related Problems}

In this section, we discuss a number of problems related to 2-MDFA minimization. While pertinent to the main problem discussed in this thesis, these state optimization problems are also interesting in their own right.

We first consider the problem of reducing the states in a DFA by converting it into a 2MDFA and establish its complexity.

Lemma 5.1 There exists no polynomial time algorithm for the problem of converting a DFA $M_{d}$ to a minimal 2-MDFA $M_{t}$, unless $\mathrm{P}=\mathrm{NP}$.

Proof. Assume that there exists an algorithm $\mathcal{A}$, that takes as input an arbitrary DFA $M_{0}$ and returns the optimal state state 2-MDFA, representing $L\left(M_{0}\right)$ in polynomial time. We can use $\mathcal{A}$ to obtain a polynomial time algorithm for the 2-MDFA state minimization problem as follows: Given an arbitrary 2-MDFA $M_{1}$, convert it into a DFA $M_{d 1}$ in polynomial time (as discussed in Section 4), and then provide it as input to Algorithm $\mathcal{A}$ to compute the optimal state 2-MDFA for the same language. However, this would mean that $\mathrm{P}=\mathrm{NP}$, since the 2-MDFA minimization problem is $\mathrm{NP}$-complete.

We now consider the Optimal Splitting and Optimal Merging problems.

(a) Optimal Splitting: Assume that we are given a DFA $M_{0}$, which is the minimal DFA for the language $L\left(M_{0}\right)$ and a number $K$. The Optimal Splitting Problem is concerned with splitting $L\left(M_{0}\right)$ into two languages $L\left(M_{1}\right)$ and $L\left(M_{2}\right)$, represented by minimal DFAs $M_{1}$ and $M_{2}$ respectively, such that $\left|Q_{M_{1}}+\right| Q_{M_{2}} \mid \leq K$. This problem is neither known to 
be NP-complete nor known to have a constant factor approximation. The optimal split number $\operatorname{split}_{M_{0}}$ for a given minimal DFA $M_{0}$ is the smallest value of $K$ such that $L\left(M_{0}\right)$ can be split into two distinct languages, $L_{1}$ and $L_{2}$.

(b) Optimal Pairing: Assume that we are given two DFAs, $M_{1}$ and $M_{2}$, representing the languages $L\left(M_{1}\right)$ and $L\left(M_{2}\right)$, respectively, and a number $K$. In the Optimal Pairing Problem, the goal is to find two new DFA's $M_{1}^{\prime}$ and $M_{2}^{\prime}$ such that:

(a) $L\left(M_{1}\right)=L\left(M_{1}^{\prime}\right)$.

(b) $L\left(M_{2}\right)=L\left(M_{2}^{\prime}\right)$.

(c) When equivalent states are merged across $M_{1}^{\prime}$ and $M_{2}^{\prime}$, the total number of states in the resultant 2-MDFA is at most $K$.

It is important to note that minimizing $M_{1}$ and $M_{2}$ does not necessarily provide the optimal solution. This is because the sub-optimal DFAs of two languages may have more states in common than their corresponding minimal versions. 


\section{Chapter 6}

\section{Implementation Results}

In this section, we discuss our empirical observations on the effectiveness of Algorithm 4.1 from the perspective of 2-MDFA minimization.

\subsection{Experimental Setup}

2-MDFAs were represented as graph data structures; for instance, see [17]. For the sake of uniformity in the comparions, all automata had exactly 200 states in our experiments. There were two probabilities associated with each 2-MDFA instance:

(i) The finality probability, $P_{f}$ - Attached to each state of the automaton, this measure represents the probability that the state is a final state.

(ii) The transition probability, $P_{t}$ - This measure represents the probability that a given transition from a state exists. For instance, if it is determined that there exists a transition on input 0 from a given state, then the said transition is equally likely to move the automaton from the given state to any state other than the dead state. If it is determined that no transition exists from a given state on a given input, then a transition to the dead state is inserted.

In our experiments, we generated three types of 2-MDFA instances: dense automata, sparse automata, and intermediate automata. Dense automata were generated with $P_{t}=1$ and $P_{f}=$ $\frac{1}{2}$; sparse automata were generated with $P_{t}=\frac{1}{2}$ and $P_{f}=\frac{1}{2}$; and, finally, intermediate automata were generated with $P_{t}=\frac{4}{5}$ and $P_{f}=\frac{1}{2}$. Random instances of 2-MDFAs were generated using 


\begin{tabular}{|c|c|c|c|}
\hline$\left|Q_{M_{0}}\right|$ (input) & $\left|Q_{M_{s}}\right|$ & $\left|Q_{M_{4}}\right|$ & $\left|Q_{M_{\text {out }}}\right|$ (output) \\
\hline 200 & 154 & 7533 & 154 \\
\hline 200 & 170 & 10178 & 170 \\
\hline 200 & 161 & 9152 & 161 \\
\hline 200 & 164 & 9396 & 164 \\
\hline 200 & 152 & 7670 & 152 \\
\hline 200 & 148 & 7866 & 148 \\
\hline 200 & 168 & 9895 & 168 \\
\hline 200 & 163 & 8440 & 163 \\
\hline 200 & 158 & 8613 & 158 \\
\hline 200 & 159 & 7090 & 159 \\
\hline
\end{tabular}

Table 6.1: Implementation profile over dense automata

both a linear congruential generator (LCG) and an inversive congruential generator (ICG). For each class of 2-MDFAs, the first five results were obtained with the LCG, and the remaining five were obtained using the ICG. The lack of discrepancy between the results from the two generators allows us to gain confidence that these generators are indeed a good approximation of true randomness, at least as far as these results are concerned.

\subsection{Observations on Dense Automata}

For our first test, we generated random instances of dense automata; i.e. automata that have a high expected number of transitions. In each instance, the pruning procedure was much more effective than the DFA conversion; in particular, the pruning procedure produced automata with at most 170 states, whereas the DFA minimization can easily produce automata with over 10000 states (see Table 6.1). As opposed to the standard representation of a regular language (a minimal DFA), Algorithm 4.1 produces a much smaller output. 


\begin{tabular}{|c|c|c|c|}
\hline$\left|Q_{M_{0}}\right|$ (input) & $\left|Q_{M_{s}}\right|$ & $\left|Q_{M_{4}}\right|$ & $\left|Q_{M_{\text {out }}}\right|$ (output) \\
\hline 200 & 2 & 2 & 2 \\
\hline 200 & 25 & 24 & 24 \\
\hline 200 & 15 & 15 & 15 \\
\hline 200 & 2 & 2 & 2 \\
\hline 200 & 2 & 2 & 2 \\
\hline 200 & 5 & 5 & 5 \\
\hline 200 & 16 & 16 & 16 \\
\hline 200 & 40 & 41 & 40 \\
\hline 200 & 15 & 15 & 15 \\
\hline 200 & 4 & 3 & 3 \\
\hline
\end{tabular}

Table 6.2: Implementation profile over sparse automata

\subsection{Observations on Sparse Automata}

For our second test, we generated random instances of sparse automata; i.e. automata that have a very low expected number of transitions. In this case, the pruning procedure and the DFA conversion procedure were almost exactly equal; neither was significantly better than the other (see Table 6.2). Although, in this case Algorithm 4.1 is no better than the standard representation of a regular language (a minimal DFA), it is at least no worse.

\subsection{Observations on Intermediate Automata}

For our third test, we generated random instances of intermediate automata; in this case, the expected number of transitions was between that of the dense and sparse automata. The intent of this was to generate some cases in which the pruning procedure is somewhat better but not strikingly better than the DFA conversion procedure. Instead, a more interesting result was obtained: either the pruning procedure was much better then the DFA conversion procedure or it was not significantly better than the DFA minimization procedure (see Table 6.3). It is curious that the anticipated result of the pruning procedure being somewhat superior was not obtained in any run. However, the pruning procedure was more effective overall, and Algorithm 4.1, 


\begin{tabular}{|c|c|c|c|}
\hline$\left|Q_{M_{0}}\right|$ (input) & $\left|Q_{M_{s}}\right|$ & $\left|Q_{M_{4}}\right|$ & $\left|Q_{M_{\text {out }}}\right|$ (output) \\
\hline 200 & 129 & 2806 & 129 \\
\hline 200 & 136 & 137 & 136 \\
\hline 200 & 97 & 1132 & 97 \\
\hline 200 & 104 & 1692 & 104 \\
\hline 200 & 122 & 123 & 122 \\
\hline 200 & 117 & 2378 & 117 \\
\hline 200 & 131 & 137 & 131 \\
\hline 200 & 114 & 2099 & 114 \\
\hline 200 & 149 & 6002 & 149 \\
\hline 200 & 109 & 1690 & 109 \\
\hline
\end{tabular}

Table 6.3: Implementation profile over intermediate automata

in many cases, produces a much smaller output than the standard representation of a regular languge (a minimal DFA). 


\section{Chapter 7}

\section{Conclusions}

Our main result is an approximation algorithm to the problem of state minimization for an arbitrary 2-MDFA. This algorithm is bounded by a linear factor and is guaranteed to have an output no larger than any equivalent DFA. This is the first non-trivial, positive result that we are aware of, insofar as approximation algorithms for NP-Hard optimization problems in Automata Theory are concerned. As part of our analysis, we showed that 2-MDFAs are only polynomially as succinct as DFAs, with respect to representing a given regular language. We established that our approximation algorithm is tight; we have shown there exist regular languages for which the state complexity of the output automaton is larger by no less than a linear factor from the state complexity of the optimal 2-MDFA. We also proposed some interesting problems with unknown computational complexity which are related to 2-MDFA minimization.

Hopefully, this result will stimulate more interest in approximation algorithms for hard minimization problems in automata theory. Towards this end, we propose two open problems that are good candidates for a further extension of our results.

(a) Is there a fast, constant factor approximation algorithm for $\mathbf{P}_{\mathbf{1}}$ ? - The existence of such an algorithm has enormous practical significance. An alternative line of research is to establish that such an algorithm cannot exist unless $P=N P$.

(b) How much savings does our algorithm provide on practical instances? - The implementation profile in Section 6 was derived using random instances; an empirical study over practical instances would serve as a dependable baseline for future empirical studies. 


\section{References}

[1] A. Meyer and M. Fischer, "Economies of description by automata, grammars, and formal systems," in Proceedings of the 12th SWAT (Annual Symposium on Switching and Automata Theory), 1971, pp. 188-191.

[2] T. Jiang and B. Ravikumar, "Minimal NFA problems are hard," in Proceedings of the 18th International Colloquium on Automata, Languages and Programming, ICALP'91 (Madrid, Spain, July 8-12, 1991), ser. LNCS, J. L. Albert and M. R. Artalejo, Eds. Berlin-Göttingen-Heidelberg-New York: Springer-Verlag, 1991, vol. 510, pp. 629-640.

[3] A. Malcher, "Minimizing finite automata is computationally hard," Theoretical Computer Science, vol. 327, no. 3, pp. 375-390, Nov. 2004.

[4] J. E. Hopcroft, "An $n \log n$ algorithm for minimizing the states in a finite-automaton," in Theory of Machines and Computations, Z. Kohavi, Ed. Academic Press, 1971, pp. 189-196.

[5] K. C. Louden, Programming Languages: Principles and Practice. Brooks/Cole, 2002.

[6] C. L. A. Clarke and G. V. Cormack, "On the use of regular expressions for searching text," ACM Trans. Program. Lang. Syst., vol. 19, no. 3, pp. 413-426, 1997.

[7] R. A. Baeza-Yates and G. H. Gonnet, "Fast text searching for regular expressions or automaton searching on tries," J. ACM, vol. 43, no. 6, pp. 915-936, 1996.

[8] T. Jiang and B. Ravikumar, "Minimal nfa problems are hard," SIAM J. Comput., vol. 22, no. 6, pp. 1117-1141, 1993.

[9] S. Yu, Q. Zhuang, and K. Salomaa, "The state complexities of some basic operations on regular languages." Theor. Comput. Sci., vol. 125, no. 2, pp. 315-328, 1994.

[10] E. L. Leiss, "Succint representation of regular languages by boolean automata." Theor. Comput. Sci., vol. 13, pp. 323-330, 1981.

[11] Meyer and Fischer, "Economy of description by automata, grammars, and formal systems," in FOCS: IEEE Symposium on Foundations of Computer Science (FOCS), 1971.

[12] B. Ravikumar and O. H. Ibarra, "Relating the type of ambiguity of finite automata to the succinctness of their representation." SIAM J. Comput., vol. 18, no. 6, pp. 1263-1282, 1989. 
[13] D. Hochbaum, Ed., Approximation Algorithms for NP-Hard Problems. Boston, Masachusetts: PWS Publishing Company, 1996.

[14] H. U. Simon, "On approximate solution for combinatorial optimization problems," SIAM Journal of Discrete Mathematics, vol. 3, pp. 294-310, 1990.

[15] P. Crescenzi and V. Kann, "Approximation compendium," http://www.nada.kth.se/ rviggo/wwwcompendium/node242.html.

[16] J. E. Hopcroft, R. Motwani, and J. D. Ullman, “Introduction to Automata Theory, Language, and Computation”, 2nd ed. Addison-Wesley, 2001.

[17] T. H. Cormen, C. E. Leiserson, and R. L. Rivest, Introduction to Algorithms, 2nd ed. Boston, Massachusetts: MIT Press and McGraw-Hill Book Company, 1992. 